\title{
resampy: efficient sample rate conversion in Python
}

\section{Brian McFee ${ }^{1}$}

DOI: $10.21105 /$ joss. 00125

1 New York University

\section{Software}

- Review ¿

- Repository ¿

- Archive ${ }^{\top}$

\section{Licence}

Authors of JOSS papers retain copyright and release the work under a Creative Commons Attribution 4.0 International License (CC-BY).

\section{Summary}

This package implements efficient, high-quality sample rate conversion using the bandlimited sinc interpolation method (Smith 2002). This method supports fast sample rate conversion for long and multi-channel signals. Its functionality and features are similar to libsamplerate (Castro 2005), but it differs by having a permissive (BSD-style) license, and it is written in Cython so that it is easier to install and use within larger Python projects.

\section{References}

Castro, Erik de. 2005. "Secret Rabbit Code (Aka Libsamplerate)(software)."

Smith, Julius O. 2002. Digital Audio Resampling Home Page. https://ccrma.stanford.edu/ jos/resar 